\title{
Español y dariya en Ceuta. Análisis y propuestas de mejora de las habilidades comunicativas en la L2 del alumnado dariyaparlante
}

Manuel José López Ruiz - Universidad de Granada

Ana María Pino Rodríguez - Ministerio de Educación y Formación Profesional

0000-0001-8261-9832

0000-0002-8110-3380

Recepción: 04.03.2021 | Aceptado: 08.03.2021

Correspondencia a través de ORCID: Ana María Pino Rodríguez

0000-0002-8110-3380

Citar: López Ruiz, MJ y Pino Rodríguez, AM (2021). Español y dariya en Ceuta. Análisis y propuestas de mejora de las habilidades comunicativas en la L2 del alumnado dariyaparlante. REIDOCREA, 10(17), 1-10.

Resumen: Este artículo analiza, a través de un enfoque descriptivo-explicativo y dentro de la investigación no experimental, el contacto que el alumnado de la minoría mayoritaria dariyaparlante de la Ciudad Autónoma de Ceuta mantiene con la lengua castellana en horario extraescolar. Teniendo presente que la adquisición y el aprendizaje de idiomas puede tener lugar en contextos educativos formales, no formales e informales, para la recogida de datos se plantea un procedimiento que permite registrar el porcentaje de estudiantes de Educación Primaria de cada grupo lingüístico en los centros educativos ceutíes, así como obtener información relativa al contacto que el alumnado ceutí dariyófono mantiene con el español como L2 fuera de la escuela. El tratamiento estadístico empleado revela que las necesidades comunicativas del uso del castellano de la muestra aludida son escasas, lo cual se convierte en un elemento determinante en el desarrollo de las competencias comunicativo-lingüísticas en español por parte de este importante sector de la población.

Palabra clave: Segunda lengua

Spanish and Dariya in Ceuta. Analysis and proposals por improving L2 capatilities of Dariya speaking students

Abstract: By means of a descriptive and explanatory approach and by means of non-experimental research, this paper analyses the contact that students from the minority Dariya-speaking community in Ceuta keep with the Spanish language outside of school hours. Keeping in mind that language acquisition and learning can take place in formal, non-formal and informal contexts, in order to collect data a procedure is established which allows the recording of the percentage of students in Primary Education from each linguistic group in Ceuta schools, as well as information related to the contact kept by Dariya-speaking students with Spanish as a Second Language outside of school. Statistical analysis undertaken shows that there is little need to communicate in Spanish for the aforementioned sample group, which inhibits an optimal development of communicative and linguistic competences in Spanish for an important sector of society.

Keyword: Second Language

\section{Introducción}

Las lenguas constituyen, sin duda, herramientas de comunicación y elementos socialmente integradores y de convivencia, que generan paisajes lingüísticos singulares propios de la sociedad multicultural y multilingüe en la cual vivimos (Hernández García y Villalba Martínez, 2004; Roa, 2006; Cenoz y Gorter, 2008). Tal diversidad lingüística suele ir de la mano de manifestaciones que, en el caso de Ceuta, representan, entre otras, a comunidades de origen español peninsular, judío sefardí, romaní, hindú, chino, marroquí y del África Subsahariana, que vienen a evidenciar la multiculturalidad transfronteriza de lugares de paso, encuentro y comunicación entre continentes (López, 2006; González y Trujillo, 2018).

En esta línea, el paisaje lingüístico de Ceuta está representado por lenguas vinculadas a grupos culturales diversos, pero, entre las mismas, junto al español destaca el dariya o árabe ceutí. En efecto, esta última lengua es utilizada por un $62,9 \%$ de la ciudadanía ceutí como lengua habitual (Lázaro, Rontomé y Cantón, 2014) y está vinculada a la 
alternancia de código o code-switching, que siendo considerada una estrategia comunicativa propia de zonas fronterizas, ha sido identificada como un elemento diferenciador del colectivo árabe ceutí, ya que viene a destacar su singularidad respecto a los no musulmanes españoles y a los musulmanes marroquíes (López, 2006). Como muestra de su importante presencia en la ciudad, destacamos que hace ya una década un $70 \%$ del alumnado que iniciaba la Educación Infantil en Ceuta lo empleaba como L1. Todo ello, hace posible referirse al mosaico lingüístico de Ceuta como un escenario propio de bilingüismo social, que también se ha categorizado como sustractivo o de pérdida, adscrito o temprano, bicultural, secundario y, en ocasiones, equilibrado (Cenoz y Gorter, 2008; Klee y Lynch, 2009).

Tal singularidad no está exenta de complejidades, aunque apenas mide $20 \mathrm{~km}^{2}$, Ceuta cuenta aproximadamente con 85.000 habitantes y presenta una densidad de población muy superior a la densidad de población del resto de España y de sus Comunidades Autónomas, con 4.242 habitantes $/ \mathrm{km}^{2}$ frente a otras con 93 personas $/ \mathrm{km}^{2}$. Con un $20,4 \%$, ostenta uno de los porcentajes de población infantil más altos del país. Actualmente, de hecho, su tasa de natalidad es la segunda del territorio nacional, $11,44 \%$. Su tasa de desempleo, según la EPA, también es muy elevada (24,6\%). (Secretaria General Técnica, 2019)

Datos internacionales y estudios nacionales han situado los resultados académicos obtenidos por parte del alumnado residente en la Ciudad Autónoma de Ceuta en las posiciones más bajas respecto a las medias españolas y europeas. Por ello, el Consejo Escolar del Estado y el Ministerio de Educación y Formación Profesional de España, así como diferentes estudios académicos han puesto sus ojos sobre los bajos niveles de los indicadores educativos de Ceuta, donde la mitad del alumnado que debería terminar el tramo de Educación Secundaria Obligatoria no llega a finalizar esta etapa. A la caída de estas tasas de escolaridad (Tablas 1 y 2 ) se suma el alto porcentaje "de repetidores al final de la etapa obligatoria", el cual "está muy cerca del $50 \%$ de estudiantes," mientras que la media española es del $29 \%$. Esta tasa de fracaso escolar, además, se concentra, fundamentalmente, sobre el alumnado dariyaparlante de la ciudad. Las causas que explican esta situación son complejas y están ligadas a aspectos que van más allá de lo puramente lingüístico y que entran en lo sociocultural y económico, posicionando a Ceuta, junto con Melilla, en el último puesto en idoneidad o correspondencia óptima entre edad y curso, al destacar por sus altos niveles de abandono escolar y alejándose de las medias educativas del resto de España, Europa y la OCDE, en su conjunto. (Ramírez, 1995; Roa, 2006; Sánchez, García y Cotrina, 2011; Secretaria General Técnica, 2016, 2017, 2019)

Tabla 1. Porcentaje de estudiantes ceutíes distribuidos según las Enseñanzas cursadas y la titularidad de los centros de matriculación. (Informe 2019 sobre el Estado del Sistema Educativo en Ceuta y Melilla. MEYFP)

\begin{tabular}{lll}
\hline Enseñanzas no universitarias & Tipos de enseñanzas & Estudiantes matriculados \\
\hline & Enseñanza pública & $80,55 \%$ \\
20.306 estudiantes & Enseñanza concertada & $18,72 \%$ \\
& Enseñanza privada & $0,73 \%$ \\
\hline
\end{tabular}

Tabla 2. Alumnado matriculado en centros ceutíes en el curso 2019-2020. (Informe 2019 sobre el Estado del Sistema Educativo en Ceuta y Melilla. Curso 2017-2018. MEYFP, 2019)

\begin{tabular}{ccclll}
\hline EI & EP & ESO & Bachillerato & FP Básica, Grado Medio y Superior & Total \\
\hline 3439 & 7653 & 4654 & 1469 & 2453 & 20.299 \\
\hline
\end{tabular}




\section{Objetivo}

Analizar la interrelación entre castellano y dariya, a través de las actividades que realiza el alumnado ceutí dariyaparlante fuera del horario escolar.

\section{Método}

La metodología empleada en esta investigación se encuadra en el enfoque descriptivoexplicativo y en la investigación no experimental. A pie de calle, sitúa su punto de partida en la solicitud a la Dirección Provincial de Educación de Ceuta de los permisos pertinentes para acceder a los centros educativos bajo su responsabilidad. El procedimiento continúa con la información a los claustros y consejos escolares de los centros educativos de la ciudad. A los mismos se les da a conocer el contenido y los objetivos de la investigación, así como los posibles beneficios que previsiblemente pueden derivarse de la misma. Además, se solicita la colaboración del profesorado y también la de las familias, a quienes se invita a participar en reuniones de carácter informativo-explicativo para que conozcan en qué consiste la investigación y cuál es su finalidad. Sobre el papel y más tarde sobre el campo de trabajo, el diseño del estudio parte de un doble procedimiento: el primero, permite, por un lado, el acceso a la población escolarizada en Educación Infantil, Primaria y Secundaria y, por otro, el registro de su lengua materna (Tabla 3), siendo estos datos de 2010, -hasta la fecha, no se ha hecho pública por parte de la Administración Educativa una actualización de estos-. Así, queda en evidencia una concentración del alumnado en función de dos grupos lingüísticos principales.

Tabla 3. Distribución del alumnado dariya-parlante matriculado en centros ceutíes

\begin{tabular}{|c|c|c|}
\hline Centros educativos ${ }^{*}$ ceutíes & Zona de influencia & Alumnado dariya-parlante \\
\hline CEIP A & 22 & $100 \%$ \\
\hline CEIP B & 18 & $100 \%$ \\
\hline CEIP C & 19 & $99,83 \%$ \\
\hline CEIP D & 15 & $97,26 \%$ \\
\hline CEIP E & 1 & $94,98 \%$ \\
\hline CEIP F & 16 & $95,65 \%$ \\
\hline CEIP G & 14 & $86,38 \%$ \\
\hline CEIP H & 20 & $83,46 \%$ \\
\hline $\mathrm{CCA}$ & 15 & $69,30 \%$ \\
\hline CEIP I & 6 & $66,76 \%$ \\
\hline CEIP J & 20 & $66,20 \%$ \\
\hline CEIP K & 10 & $62,78 \%$ \\
\hline CEIP L & 13 & $50,24 \%$ \\
\hline CEIP M & 2 & $48,47 \%$ \\
\hline CEIP N & 8 & $42,99 \%$ \\
\hline CEIP N & 9 & $41,87 \%$ \\
\hline CEIP 16 & 12 & $23,34 \%$ \\
\hline CCB & 8 & $22,64 \%$ \\
\hline
\end{tabular}




\begin{tabular}{|c|c|c|}
\hline $\mathrm{CCC}$ & 2 & $21,54 \%$ \\
\hline CCD & 2 & $6,88 \%$ \\
\hline
\end{tabular}

Efectivamente, los porcentajes de estudiantes atribuidos a centros de la red pública y concertada de Ceuta son dispares y, combinados con el uso del dariya entre sus estudiantes, dan lugar a una foto fija dentro de la cual un $92.16 \%$ del total del alumnado dariyaparlante de la Etapa de Infantil está escolarizado en centros públicos, mientras que un $7.84 \%$ lo está en centros concertados. Mientras tanto, los porcentajes de uso del dariya y del castellano como lengua materna por parte del alumnado ceutí en Educación Primaria evidencian que un $89,87 \%$ del total del alumnado dariyaparlante está escolarizado en centros públicos, siendo un $10,13 \%$ el adscrito a centros concertados.

Asimismo, se establece sobre nuestro universo una muestra cuyos criterios de selección son los siguientes:

- finalizar la Primera Etapa de la Enseñanza Obligatoria después de haber cursado, al menos, 9 años de escolaridad;

- haber sido inscrita o inscrito en un Instituto de Educación Secundaria.

De este modo, la muestra queda conformada por la totalidad de los estudiantes de Sexto de Primaria, que, después de haber podido pasar nueve cursos escolares dentro del sistema educativo, se encuentra en el momento de hacer la transición a la Etapa de Educación Secundaria Obligatoria. La misma consta de 1436 sujetos.

Se procede a pasar a dicha muestra un cuestionario (López, 2006) en las primeras semanas del curso en el cual se accede al Primer Curso de la ESO en los diferentes centros de Secundaria públicos y concertados de la ciudad. Los elementos de este instrumento quedan recogidos de forma abreviada en la Tabla 4, donde se aprecian distintas preguntas agrupadas en dos bloques: I. Actividades; II. Intencionalidad. El primero de estos bloques está orientado a describir las actividades extraescolares realizadas por el alumnado dariyaparlante a través de los siguientes ítems: tipo de actividad extraescolar; lugar donde se realiza; motivo de su realización; día o días de la semana a lo largo de los cuales se efectúa; carga horaria de dedicación a la actividad; y fecha de su comienzo. El segundo bloque considera la intencionalidad del alumnado, las familias, el profesorado y la propia administración educativa con relación a la oferta y realización de dichas actividades.

Tabla 4. Elementos básicos del Cuestionario. (López, 2006)

\begin{tabular}{|c|c|c|c|c|}
\hline \multicolumn{5}{|c|}{ Cuestionario de ocupación del tiempo fuera del centro } \\
\hline Centro & Etapa & Curso & Grupo & Código \\
\hline Ítem 1 & \multicolumn{4}{|c|}{ Actividades realizadas por las tardes o en el fin de semana } \\
\hline Ítem 2 & \multicolumn{4}{|c|}{ Lugar donde se realizan } \\
\hline Ítem 3 & \multicolumn{4}{|c|}{ Motivo por el cual se realizan } \\
\hline
\end{tabular}


$\begin{array}{ll}\text { Ítem } 4 & \text { Carácter gratuito o de pago } \\ \text { Ítem } 5 & \text { Días de la semana en los que se realizan } \\ \text { Ítem } 6 & N^{0} \text { de horas invertidas en su realización } \\ \text { Ítem } 7 & \text { Año de inicio de esas actividades } \\ \text { Ítem } 8 & \text { Interés por participar en un programa de aaee en el centro educativo de referencia } \\ \text { Ítem } 9 & \text { Tipo de actividades en las que se participaría dentro de ese programa }\end{array}$

La recogida de información mediante los cuestionarios es llevada a cabo voluntariamente por docentes y trabajadores sociales de los centros educativos, bien durante los periodos de recreo, bien en otros momentos de la jornada escolar y estudiante por estudiante.

\section{Resultados}

Los datos relativos al contexto extraescolar quedan organizados en torno a la cantidad, el tipo, la frecuencia y la carga horaria de las actividades extraescolares realizadas por parte del alumnado que constituye nuestra muestra, arrojando los datos que se presentan a continuación.

Con respecto a la cantidad, un $24,70 \%$ de la muestra no dedica el tiempo que pasa fuera de la escuela a ninguna actividad extraescolar. De este modo, el alumnado que sí las realiza constituye un $75,30 \%$ de la misma. Estos datos quedan recogidos en la Tabla 5.

\begin{tabular}{ccc}
\multicolumn{2}{c}{ Tabla 5. Actividades extraescolares realizadas por el alumnado ceutí } \\
\hline Frecuencia & Porcentaje & Válidos \\
\hline 354 & 24.7 & NO \\
1082 & 75.3 & Sí \\
1436 & 100 &
\end{tabular}

En cuanto al número de actividades realizadas (Tabla 6), destaca el grupo que realiza una sola actividad, con un $41,40 \%$, seguido por el que realiza dos actividades, con un $20,3 \%$. Las tres actividades se corresponden con un $9,5 \%$ del alumnado, y las 4,5 ó más, solo de un $4,3 \%$ del conjunto.

Tabla 6. Número de actividades extraescolares realizadas por el alumnado ceutí

\begin{tabular}{ccccccc}
\hline $\mathbf{0}$ & $\mathbf{1}$ & $\mathbf{2}$ & $\mathbf{3}$ & $\mathbf{4}$ & $\mathbf{5}$ & MÁS DE 5 \\
\hline 355 & 594 & 291 & 136 & 31 & 18 & 11 \\
24.7 & 41,4 & 20,3 & 9,5 & 2,2 & 1,3 & 8 \\
24.7 & 41,4 & 20,3 & 9,5 & 2,2 & 1,3 & 8 \\
24.7 & 66,1 & 86,4 & 95,8 & 98,0 & 99,2 & 100,0 \\
\hline
\end{tabular}


Un análisis diferencial de las actividades extraescolares realizadas en relación con la cultura del universo muestra que un $77,01 \%$ de los estudiantes dariyaparlantes ocupa parte de su tiempo extraescolar realizando actividades extraescolares, mientras que entre los no dariyófonos este porcentaje es de $73,71 \%$ (Tabla 7 ). Los datos sobre porcentajes en relación con el número de AAEE realizadas por cada grupo cultural se pueden ver en las Tablas 7 y 8 . Los análisis pertinentes no muestran diferencias significativas en relación con la vinculación entre el ítem y la ocupación del tiempo extraescolar. Del mismo modo, el análisis realizado sobre el número de actividades extraescolares en relación con la cultura tampoco ofrece diferencias significativas.

Tabla 7. Actividades extraescolares y cultura del alumnado.

\begin{tabular}{ccccc}
\hline Actividades & Alumnado con L1 dariya & Alumnado con L1 español & Alumnado con otra lengua materna & Total \\
\hline No realiza & 163 & 189 & 12 & 352 \\
Sí realiza & 546 & 526 & 1072 \\
& & & 1424 \\
\hline
\end{tabular}

Tabla 8. Relación entre el número de actividades extraescolares realizadas y los dos grupos culturales mayoritarios de Ceuta.

\begin{tabular}{cccc}
\hline Número de AAEE & Alumnado con lengua materna dariya & Alumnado con lengua materna distinta del dariya & Total \\
\hline 0 & 163 & 189 & 352 \\
1 & 306 & 284 & 590 \\
2 & 147 & 139 & 286 \\
3 & 65 & 71 & 136 \\
4 & 14 & 17 & 31 \\
5 & 10 & 8 & 18 \\
Más de 5 & 4 & 7 & 11 \\
\hline
\end{tabular}

En cuanto al tipo de actividades, las más comúnmente realizadas (Gráfico 1), por orden de frecuencia, son:

- Clases particulares, con un $41,13 \%$

- Actividades deportivas, con un $40,94 \%$

- Escuelas coránicas, con un 30,04\%

- Clases de Idioma "dariya", con un 19,50\%

La asistencia a estos cuatro tipos de actividades extraescolares por parte del alumnado dariyaparlante supone un escaso contacto con sus pares castellanoparlantes, ya que se realizan junto a otros niños y otras niñas de la misma lengua, debiéndose ello a que las clases particulares y las actividades deportivas se celebran en el centro educativo de pertenencia, cuya población se caracteriza por los condicionamientos lingüísticos ya expuestos en relación con la distribución del alumnado. La asistencia a escuelas coránicas tiene lugar en mezquitas y la asistencia a clases de árabe, también. 


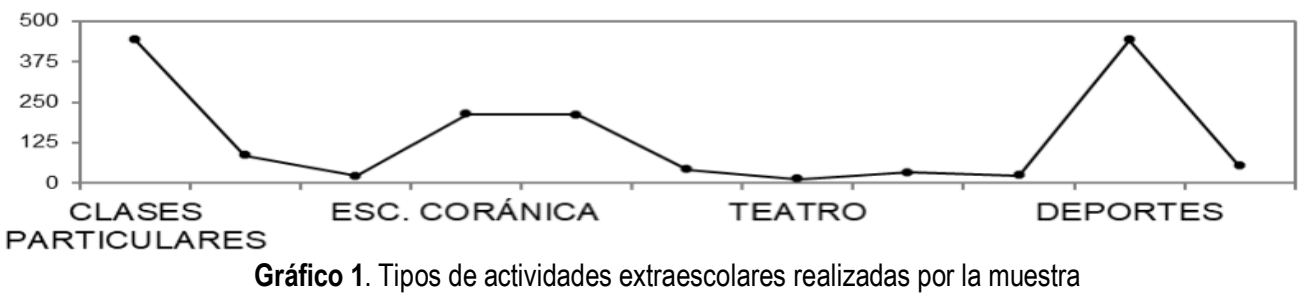

La frecuencia con la que el alumnado realiza estas actividades extraescolares (Gráfico 2) destaca los cuatro tipos de actividades más concurridos. En relación con el primer tipo (clases particulares), con un $41,13 \%$ del alumnado, los casos más frecuentados de asistencia son:

- 5 días semanales, $53,71 \%$

- 4 días semanales, $28,31 \%$

- 1 día semanal, $11,68 \%$

Las actividades deportivas representan la segunda actividad más concurrida, con un $40,94 \%$, siendo los casos más frecuentados de asistencia los siguientes:

- 4 días semanales, $13,54 \%$

- 3 días semanales, $25,51 \%$

- 2 días semanales, $29,11 \%$

La tercera actividad con mayor índice de realización es la asistencia a escuelas coránicas, con un $30,04 \%$ del total de alumnado de cultura musulmana, siendo los tres casos más frecuentados estos:

- 6 días semanales, $7,98 \%$

- 5 días semanales, $45,70 \%$

- 4 días semanales, $45,70 \%$

La cuarta actividad más realizada es la asistencia a clases de idiomas, con un $19,50 \%$, resultando los siguientes los casos más frecuentados:

- 5 días semanales, con un $16,11 \%$

- 3 días semanales, con un $55,45 \%$

- 1 día semanal, con un $29,38 \%$

Como puede apreciarse, la actividad extraescolar que ocupa mayor número de días de la semana es la asistencia a escuelas coránicas.

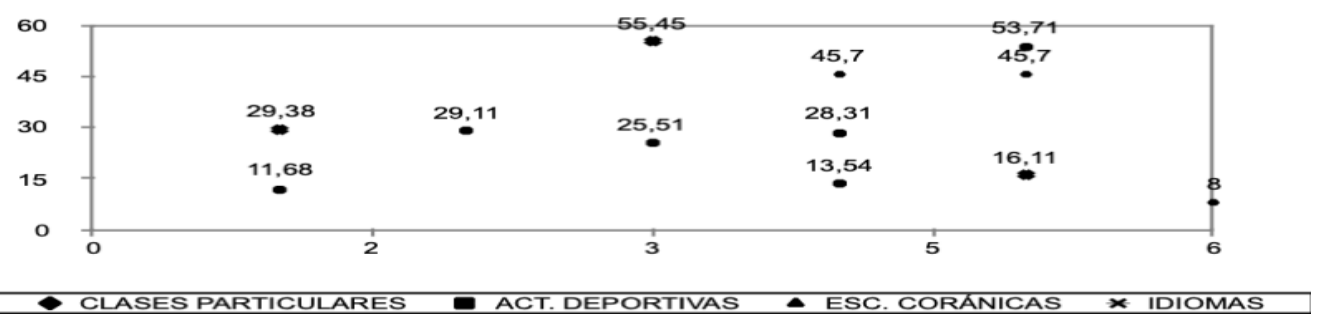

Gráfico 2. Número de días de la semana en los que se realizan las actividades más frecuentadas. 
El alumnado que realiza actividades extraescolares dedica una media de $8 \mathrm{~h}$ cada semana a las mismas. El análisis pormenorizado de los datos revela una diferencia horaria significativa en relación con el tiempo dedicado a cada tipo de actividad. Así, el análisis comparativo entre las cuatro actividades extraescolares más frecuentadas deja ver los siguientes datos:

- El $41,13 \%$ del alumnado que realiza actividades extraescolares, pasa 5,39 h semanales a clases particulares.

- El $40,94 \%$ del alumnado que realiza actividades extraescolares, dedica 5,48 h semanales a las actividades deportivas.

- El 30,04\% del alumnado de cultura musulmana que asiste a escuelas coránicas, lo hace con una media de $9,41 \mathrm{~h}$ semanales.

- El $19,50 \%$ del alumnado que realiza actividades extraescolares, asiste a clases de idiomas $12,59 \mathrm{~h}$ semanales.

Como puede observarse, la asistencia a clases de idiomas es la actividad que ocupa mayor número de horas. Un $67,30 \%$ del alumnado que ha marcado esta opción acude a las escuelas coránicas para aprender árabe clásico.

\section{Discusión}

Dados estos resultados, se aprecia que el contexto social dentro del cual se desarrollan las actividades extraescolares de los estudiantes ceutíes dariyaparlantes no favorece la adquisición y el aprendizaje del español como L2. Así, los datos presentados sobre el contacto dariya-español que mantiene el alumnado arabófono de los centros educativos ceutíes en contextos extraescolares vienen a evidenciar las escasas oportunidades de uso del castellano como L2 para este grupo. Si unimos esta escasez de contacto con la importancia que tiene el contexto social dentro del cual se desarrollan esas actividades extraescolares y con la importancia de estas en tanto que elemento determinante en la adquisición y el aprendizaje del español como L2, este alumnado se encuentra en una situación de riesgo muy considerable.

Tales hallazgos corroboran las conclusiones obtenidas por López, Serrano y Vila (2004) según los cuales los ambientes de exposición al español a los que se somete el alumnado de Ceuta están muy restringidos tanto a nivel familiar, como a nivel social. Ello dificulta la mejora y consolidación de las habilidades comunicativas en español, dado que estos avances se producen a través de interacciones habituales, significativas y útiles que, si bien pueden tener lugar en contextos de aprendizaje formal, como los propios de la escuela, también se generan en situaciones de educación informal y de aprendizaje no formal, como las surgidas en esos otros contextos extraescolares y junto a otros agentes, distintos al profesorado, como son la familia, la pandilla, los medios de comunicación, las congregaciones religiosas o los monitores de ocio y tiempo libre.

Así las cosas, es necesario facilitar a los estudiantes oportunidades de uso del español en intercambios comunicativos diversos, resultando la interacción entre estudiantes con L1 español y con L1 dariya una importante opción para tener en cuenta en el desarrollo de las actividades extraescolares y en otros contextos de educación no formal e informal. También lo es el uso de metodologías orientadas al desarrollo de la Competencia en Comunicación Lingüística, dado que en la adquisición y el aprendizaje de idiomas no solo es importante la cantidad de tiempo de exposición y de uso de la lengua, sino también la calidad de los intercambios lingüísticos que se produzcan en estos contextos. 
Ambas opciones constituyen recursos apropiados a los que acudir en contextos educativos formales, pero también en los no formales e informales. Por ello, realizamos las siguientes reflexiones y propuestas educativas:

1. La generalización en el empleo de metodologías comunicativas a lo largo y ancho de la Educación Infantil y de la Educación Obligatoria, Etapa de Primaria y Educación Secundaria Obligatoria, por parte de los equipos docentes. Desde los propios currículos del MEYFP y de las Administraciones educativas, apoyados en estudios de distintos campos y ámbitos de la Lingüística, la Sociolingüística y la Psicolingüística, se promulga la importancia del enfoque comunicativo y funcional en la enseñanza de la lengua, lo cual resulta especialmente relevante cuando se trabaja con niñas y niños que al ingresar en el sistema educativo utilizan exclusivamente una lengua distinta a la de la escuela o son mucho más competentes en esa lengua que en español. Además, son numerosos los investigadores que alertan sobre la importancia de una rica estimulación lingüística, dado que esta predice la mayor o menor probabilidad de éxito en el aprendizaje de una segunda lengua (Pearson, 2007). Asimismo, la formación y la capacitación del profesorado de Educación Infantil y Primaria y de la ESO y del profesorado de Formación Profesional, constituyen factores de gran relevancia en el desarrollo de las habilidades comunicativas del alumnado. En relación a este último, podemos hacer alusión al profesorado de los Ciclos de Técnico Superior en Educación Infantil, en Animación Sociocultural y Turística, en Actividades Ecuestres, en Guía en el Medio Natural y de Tiempo Libre, en Acondicionamiento Físico, en Enseñanza y Animación Sociodeportiva y en Intervención Socioeducativa, dado que al desempeñar las profesiones en las cuales se han formado, tendrán ocasión de interaccionar con alumnado de distintas etapas y con distintas necesidades lingüísticas. Ciñéndonos al contexto de esta ciudad, la Facultad de Educación, Economía y Tecnología del Campus de Ceuta de la Universidad de Granada, así como la Unidad de Programas Educativos de la Dirección Provincial de Educación de Ceuta y el propio Ministerio de Educación y Formación Profesional -MEYFP-, son instituciones con un papel fundamental en este sentido, al tener las competencias necesarias para diseñar itinerarios formativos adecuados a las características del entorno. Para ello, sería útil tomar en consideración los hallazgos de Pérez-Cañado (2014) con relación a las necesidades y demandas formativas del profesorado inmerso en programas bilingües.

2. La incorporación de estrategias propias de la enseñanza de ELE, así como las vinculadas a la enseñanza de lenguas extranjeras, en general, tanto a las aulas de Infantil, como a las del resto de etapas educativas, podría aportar grandes mejoras a nivel metodológico y a nivel de resultados académicos (Pavón, 2009; Trujillo, 2005). Conceptos básicos propios del enfoque AICLE -Aprendizaje Integrado de Contenidos y Lenguas Extranjeras- como BICS y CALP -Basic Interpersonal Communicative Skills y Cognitive Academic Linguistic Proficiency-, la revisitación o revisión cíclica de contenidos, las tareas comunicativas o la interacción entre iguales a través de fórmulas presenciales o virtuales, de trabajo cooperativo y/o colaborativo, podrían jugar a favor de la consolidación y mejora del conjunto de las habilidades lingüísticas del alumnado y, nuevamente, de la cohesión social. Todo ello ha de ser contemplado a través de los planes y proyectos de centro, considerando entre ellos, por su relevancia respecto al tema que nos ocupa, y aunque no sea prescriptivo en territorio MEYFP, el Plan Lingüístico de Centro (Trujillo y Rubio, 2009; Trujillo, 2010). 


\section{Referencias}

Cenoz, J y Gorter, D (2008). El estudio del paisaje lingüístico, Hizkunea, 22, 1-8.

González, A y Trujillo, F (2018). Paisajes lingüísticos y translingüismo como propuesta lingüística a una pedagogía de frontera, Cuadernos de Pedagogía, 492, 104-108.

Hernández García, M y Villalba Martínez, F (2004). Diseño curricular para la enseñanza del español como L2 en contextos escolares. En Fortele. Documentos de Nivel I: Inicial. Murcia: Consejería de Educación y Cultura.

Jiménez, RA, Sánchez, CA, García, M y Cotrina, M (2011). Explorar y comprender el fracaso escolar en el contexto multicultural de Ceuta. Respuestas de la institución escolar. En FJ García Castaño y N Kressova (coords.), Actas del I Congreso Internacional sobre Migraciones en Andalucía (133-147). Granada: Instituto de Migraciones. Universidad de Granada.

Klee, CA y Lynch, A (2009). El español en contacto con otras lenguas. Washington DC: Georgetown University Press.

Lázaro, MI, Rontomé, C y Cantón, JM (2014). Los usos lingüísticos de la población de Ceuta: el español, el árabe y el beréber. Ceuta: Instituto de Estudios Ceutíes.

López Ruiz, MJ (2006). El perfil del alumnado de compensación educativa del C.P. Federico García Lorca, como base para el diseño y desarrollo de un programa de compensación educativa más adecuado a la realidad. Tesis Doctoral: Universidad de Granada.

López, MJ, Serrano, M y Vila, I (2004). El bilingüismo: historia y panorama actual. Programas de cambio de lengua hogarescuela. La realidad de las escuelas ceutíes. La práctica educativa de las escuelas ceutíes. En Inmigración, Interculturalidad y Convivencia III: Congreso internacional celebrado del 17 al 20 de junio de 2003 en Ceuta (127). Ceuta: Instituto de Estudios Ceutíes.

Pavón, V (2009). Cambios en la realidad educativa a través de la enseñanza integrada de lengua y contenidos. En J Jiménez Heffernan et al., Las lenguas extranjeras como vehículo de comunicación intercultural (65-84). Madrid: Secretaría General Técnica del Ministerio de Educación.

Pearson, BZ (2007). Social factors in childhood bilingualism in the United States. Applied psycholinguistics, 28(3), 399-410. https://doi.org/10.1017/S014271640707021X

Pérez-Cañado, ML (2014). Teacher Training Needs for Bilingual Education: In-service Teachers' Perceptions. International Journal of Bilingual Education and Bilingualism, 19:3, 266-295. https://doi.org/10.1080/13670050.2014.980778

Ramírez Salguero, MI (2004). La adaptación como factor de rendimiento de la población escolar de la Comunidad Musulmana Ceutí. GRANADA: IMPREMESUR.

Roa, JM (2006). Rendimiento escolar y "situación diglósica" en una muestra de escolares de educación primaria en Ceuta. Revista Electrónica de Investigación Educativa 8(1), 1-15.

Scretaria General Técnica (2019. Pisa 2018: programa para la evaluación internacional de los alumnos: informe español. Madrid: Ministerio de Educación y Formación Profesional.
Secretaria General Técnica (2016). PISA 2015: Programa para la Evaluación Internacional de los Alumnos: Informe español. Madrid: Ministerio de Educación y Formación Profesional.

Secretaría General Técnica (2017). Informe 2016 sobre el estado del sistema educativo, curso 2014-2015. Madrid: Ministerio de Educación y Formación Profesional.

Secretaría General Técnica (2019). Informe 2018 sobre el estado del sistema educativo, curso 2016-2017. Madrid: Ministerio de Educación y Formación Profesional.

Trujillo, F (2005). La integración de lenguaje y contenidos en la enseñanza del español como segunda lengua en el contexto escolar: implementación y evaluación. redELE, 4, 1-14.

Trujillo, F (2010). La competencia en comunicación lingüística como proyecto de centro retos, posibilidades y ejemplificaciones. Revista lenguaje y textos, 32, 35-40.

Trujillo, F y Rubio, R (2009). EI PLC como respuesta sistémica al reto de la competencia comunicativa en entornos educativos formales: propuesta de análisis de casos. Revista lenguaje y textos, 39, 29-38. 